\title{
Riemannian manifolds with two circulant structures
}

\author{
Iva Dokuzova
}

January 17, 2021

\begin{abstract}
We consider a three-dimensional Riemannian manifold equipped with two circulant structures - a metric $g$ and a structure $q$, which is an isometry with respect to $g$ and the third power of $q$ is minus identity. We discuss some curvature properties of this manifold, we give an example of such a manifold and find a condition for $q$ to be parallel with respect to the Riemannian connection of $g$.
\end{abstract}

Mathematics Subject Classification (2010): 53C05, 53B20

Keywords: Riemannian metric, circulant matrix, curvature properties

\section{Introduction}

Important role in the geometry of the differentiable manifolds play additional structures. Often the structure satisfies an equation of second power, for example [1], 2], 3], but rarely it satisfies an equation of another power [5], [6], [8], 9]. On the other hand the application of circulative structures in the geometry is interesting. In [7] a geometric application of circulant matrices was obtained.

In this paper we consider a three-dimensional differentiable manifold $M$ with a Riemannian metric $g$ whose matrix in local coordinates is a special circulant matrix. Moreover, we consider an additional structure $q$ on $M$ with $q^{3}=-$ id such that its matrix in local coordinates is also circulant. It turns out that $q$ is an isometry with respect to $g$. We note that there exists a local coordinate system, where the matrices of the components of these structures are circulant [4].

The paper is organized as follows. In Sect. 1 we introduce on a threedimensional differentiable manifold $M$ a Riemannian metric $g$ whose matrix in local coordinates is a special circulant matrix. Furthermore, we consider an additional structure $q$ on $M$ with $q^{3}=-$ id such that its matrix in local 
coordinates is also circulant. Thus, the structure $q$ is an isometry with respect to $g$. We denote by $(M, g, q)$ the manifold $M$ equipped with the metric $g$ and the structure $q$. In Sect. 2 we obtain the conditions under which an orthogonal basis of type $\left\{x, q x, q^{2} x\right\}$ exists in the tangent space of a manifold $(M, g, q)$. The main result there is Theorem 2.3. We give an example of such an orthogonal basis. In Sect. 3 we establish relations between the sectional curvatures of some special 2-planes in the tangent

space. In Sect. 4 we obtain a necessary and sufficient condition for $q$ to be parallel with respect to the Riemannian connection of $g$. Finally, in Sect. 5 , we give an example of the considered manifold $(M, g, q)$.

\section{Preliminaries}

Let $M$ be a three-dimensional manifold with a Riemannian metric $g$. Let the matrix of the local components of the metric $g$ at an arbitrary point $p\left(X^{1}, X^{2}, X^{3}\right) \in M$ be a circulant matrix of the following form:

$$
\left(g_{i j}\right)=\left(\begin{array}{lll}
A & B & B \\
B & A & B \\
B & B & A
\end{array}\right),
$$

where $A$ and $B$ are smooth functions of $X^{1}, X^{2}, X^{3}$. We will suppose that

$$
A>B>0 \text {. }
$$

Then the conditions to be a positive definite metric $g$ are satisfied:

$$
\begin{gathered}
A>0, \quad\left|\begin{array}{ll}
A & B \\
B & A
\end{array}\right|=(A-B)(A+B)>0, \\
\left|\begin{array}{lll}
A & B & B \\
B & A & B \\
B & B & A
\end{array}\right|=(A-B)^{2}(A+2 B)>0 .
\end{gathered}
$$

We denote by $(M, g)$ the manifold $M$ equipped with the Riemannian metric $g$ defined by (1) with conditions (2).

Let $q$ be an endomorphism in the tangent space $T_{p} M$ of the manifold $(M, g)$ with

$$
q^{3}=-\mathrm{id}, \quad q \neq-\mathrm{id},
$$


where id is the identity in $T_{p} M$. We suppose the local coordinates of $q$ are given by the circulant matrix

$$
\left(q_{i}^{j}\right)=\left(\begin{array}{lll}
a_{1} & a_{2} & a_{3} \\
a_{3} & a_{1} & a_{2} \\
a_{2} & a_{3} & a_{1}
\end{array}\right), \quad\left(a_{1}, a_{2}, a_{3} \in \mathbb{R}\right) .
$$

Due to (3) we have $\left(q_{i}^{\cdot j}\right)^{3}=-E,\left(q_{i}^{\cdot j}\right) \neq-E$ ( $E$ is the unit matrix). Then we get the following system for $a_{1}, a_{2}, a_{3}$ :

$$
\begin{aligned}
& a_{1}^{3}+a_{2}^{3}+a_{3}^{3}+6 a_{1} a_{2} a_{3}=-1, \\
& a_{1}^{2} a_{2}+a_{1} a_{3}^{2}+a_{2}^{2} a_{3}=0, \\
& a_{1} a_{2}^{2}+a_{3} a_{1}^{2}+a_{3}^{2} a_{2}=0,
\end{aligned}
$$

where $\left(a_{1}, a_{2}, a_{3}\right) \neq(-1,0,0)$. Hence we get two cases for $q$ :

$$
\left(\begin{array}{ccc}
0 & -1 & 0 \\
0 & 0 & -1 \\
-1 & 0 & 0
\end{array}\right), \quad\left(\begin{array}{ccc}
0 & 0 & -1 \\
-1 & 0 & 0 \\
0 & -1 & 0
\end{array}\right) .
$$

In the further studies of both cases similar results are obtained. We choose the case

$$
\left(q_{i}^{\cdot j}\right)=\left(\begin{array}{ccc}
0 & -1 & 0 \\
0 & 0 & -1 \\
-1 & 0 & 0
\end{array}\right) .
$$

We denote by $(M, g, q)$ the manifold $(M, g)$ equipped with the structure $q$ defined by (44).

Further, $x, y, z, u$ will stand for arbitrary elements of the algebra on the smooth vector fields on $M$ or vectors in the tangent space $T_{p} M$. The Einstein summation convention is used, the range of the summation indices being always $\{1,2,3\}$.

From (11) and (4) we get immediately the following

Proposition 1.1. The structure $q$ of the manifold $(M, g, q)$ is an isometry with respect to the metric $g$, i.e.

$$
g(q x, q y)=g(x, y) .
$$

\section{Orthogonal $q$-bases of $T_{p} M$}

Definition 2.1. A basis of type $\left\{x, q x, q^{2} x\right\}$ of $T_{p} M$ is called a $q$-basis. In this case we say that the vector $x$ induces a q-basis of $T_{p} M$. 
If $x=\left(x^{1}, x^{2}, x^{3}\right) \in T_{p} M$, then $q x=\left(-x^{2},-x^{3},-x^{1}\right), q^{2} x=\left(x^{3}, x^{1}, x^{2}\right)$. Obviously, we have the following

Proposition 2.2. A vector $x=\left(x^{1}, x^{2}, x^{3}\right)$ induces a $q$-basis of $T_{p} M$ if and only if

$$
3 x^{1} x^{2} x^{3} \neq\left(x^{1}\right)^{3}+\left(x^{2}\right)^{3}+\left(x^{3}\right)^{3} .
$$

In our next considerations we use an orthogonal $q$-basis of $T_{p} M$. That's why we will prove the existence of such bases.

Theorem 2.3. If $x=\left(x^{1}, x^{2}, x^{3}\right)$ induces a $q$-basis of $T_{p} M$, then for the angles $\varphi=\angle(x, q x), \phi=\angle\left(x, q^{2} x\right)$ and $\theta=\angle\left(q x, q^{2} x\right)$ we have

$$
\begin{gathered}
\cos \varphi=-\cos \phi=\cos \theta=-\frac{B a+(A+B) b}{A a+2 B b}, \\
\varphi \in\left(\frac{\pi}{3}, \pi\right), \phi=\theta \in\left(0, \frac{2 \pi}{3}\right),
\end{gathered}
$$

where

$$
a=\left(x^{1}\right)^{2}+\left(x^{2}\right)^{2}+\left(x^{3}\right)^{2}, b=x^{1} x^{2}+x^{1} x^{3}+x^{2} x^{3} .
$$

Proof. According to (11), (2), (4) and (5), we obtain

$$
\begin{gathered}
\cos \varphi=\frac{g(x, q x)}{g(x, x)}=-\frac{B a+(A+B) b}{A a+2 B b}, \\
\cos \phi=\frac{g\left(x, q^{2} x\right)}{g(x, x)}=\frac{B a+(A+B) b}{A a+2 B b}, \\
\cos \theta=\frac{g\left(q x, q^{2} x\right)}{g(x, x)}=-\frac{B a+(A+B) b}{A a+2 B b} .
\end{gathered}
$$

Then we have (7).

Now we prove (8). Since $\varphi, \phi$ and $\theta$ are angles between vectors, we have $\varphi, \phi, \theta \in[0, \pi]$. Let us suppose that $\phi \geq \frac{2 \pi}{3}$. Then, due to (7) and (9), we find $\left(x^{1}+x^{2}+x^{3}\right)^{2} \leq 0$, i.e. $x^{1}+x^{2}+x^{3}=0$. The last equality is in contradiction with the inequality (6) . Consequently, we have $\phi<\frac{2 \pi}{3}$. Since the vectors $x$ and $q^{2} x$ are linearly independent, we have $\phi>0$ and then $\phi \in\left(0, \frac{2 \pi}{3}\right)$, i.e. $\cos \phi \in\left(-\frac{1}{2}, 1\right)$. Thus, from (77) we get $\cos \varphi \in\left(-1, \frac{1}{2}\right)$, i.e. $\varphi \in\left(\frac{\pi}{3}, \pi\right)$.

Corollary 2.4. The following assertions are true: 
(i) If a vector $x=\left(x^{1}, x^{2}, x^{3}\right)$ induces a q-basis of $T_{p} M$, then this basis is an orthogonal q-basis if and only if

$$
B a+(A+B) b=0 .
$$

(ii) There exist orthogonal q-bases in $T_{p} M$.

Proof. The assertion (i) follows immediately from (7), and (ii) follows from (8).

Example. Let $x=(0,-(A+B)+\sqrt{(A-B)(A+3 B)}, 2 B) \in T_{p} M$. Then according to (9) we get

$$
\begin{aligned}
& a=2(A+B)((A+B)-\sqrt{(A-B)(A+3 B)}), \\
& b=-2 B((A+B)-\sqrt{(A-B)(A+3 B)}) .
\end{aligned}
$$

These values satisfy the condition (10). So $x$ induces an orthogonal $q$-basis $\left\{x, q x, q^{2} x\right\}$ of $T_{p} M$.

\section{Some curvature properties of $(M, g, q)$}

Let $\nabla$ be the Riemannian connection of the metric $g$ on $(M, g, q)$. The curvature tensor $R$ of $\nabla$ is $R(x, y) z=\nabla_{x} \nabla_{y} z-\nabla_{y} \nabla_{x} z-\nabla_{[x, y]} z$. The tensor of type $(0,4)$ associated with $R$ is defined as follows

$$
R(x, y, z, u)=g(R(x, y) z, u) .
$$

For the local components of the tensor $R$ of type $(1,3)$ we have [10]:

$$
R_{i j k}^{h}=\partial_{j} \Gamma_{i k}^{h}-\partial_{k} \Gamma_{i j}^{h}+\Gamma_{i k}^{t} \Gamma_{t j}^{h}-\Gamma_{i j}^{t} \Gamma_{t k}^{h},
$$

where the Christoffel symbols $\Gamma_{i j}^{h}$ of $\nabla$ are

$$
2 \Gamma_{i j}^{h}=g^{t h}\left(\partial_{i} g_{t j}+\partial_{j} g_{t i}-\partial_{t} g_{i j}\right) .
$$

Here $g^{i j}$ are the elements of the inverse matrix of $\left(g_{i j}\right)$.

We denote $D=\left(g_{i j}\right)$ and

$$
A_{i}=\frac{\partial A}{\partial X^{i}}, \quad B_{i}=\frac{\partial B}{\partial X^{i}},
$$

where $A$ and $B$ are the functions from (1). From (11), (11), (12) and (13) by direct calculations we obtain the following 
Proposition 3.1. The nonzero components of the curvature tensor of type $(0,4)$ of the manifold $(M, g, q)$ are

$$
\begin{aligned}
R_{1212}= & \frac{1}{2}\left(2 B_{21}-A_{11}-A_{22}\right) \\
+ & \frac{A+B}{4 D}\left(2 A_{3} B_{2}-A_{3}^{2}+\left(B_{1}-B_{2}-B_{3}\right)\left(B_{1}+B_{2}-B_{3}\right)\right) \\
- & \frac{B}{4 D}\left(2 A_{1}\left(B_{1}+B_{2}-B_{3}\right)-2 B_{2}\left(B_{1}+B_{2}-B_{3}\right)-2 A_{1} A_{3}+2 A_{3} B_{2}\right), \\
R_{1313}= & \frac{1}{2}\left(2 B_{31}-A_{11}-A_{33}\right) \\
+ & \frac{A+B}{4 D}\left(2 A_{2} B_{3}-A_{2}^{2}+\left(-B_{1}+B_{2}+B_{3}\right)\left(-B_{1}+B_{2}-B_{3}\right)\right) \\
- & \frac{B}{4 D}\left(2 A_{1}\left(B_{1}-B_{2}+B_{3}\right)-2 B_{3}\left(B_{1}-B_{2}+B_{3}\right)-2 A_{1} A_{2}+2 A_{2} B_{3}\right), \\
R_{2323}= & \frac{1}{2}\left(2 B_{23}-A_{22}-A_{33}\right) \\
+ & \frac{A+B}{4 D}\left(2 B_{3} A_{1}-A_{1}^{2}+\left(B_{1}-B_{2}+B_{3}\right)\left(B_{1}-B_{2}-B_{3}\right)\right) \\
- & \frac{B}{4 D}\left(2 A_{2}\left(B_{2}+B_{3}-B_{1}\right)+2 B_{3}\left(B_{1}-B_{2}-B_{3}\right)-2 A_{1} A_{2}+2 A_{1} B_{3}\right), \\
R_{1213}= & \frac{1}{2}\left(B_{21}+B_{31}-B_{11}-A_{23}\right) \\
& \left.+\frac{A+B}{4 D}\left(A_{1}\left(B_{2}-B_{3}+B_{1}\right)+2 B_{3}\left(-B_{1}-B_{2}+B_{3}\right)+A_{2} A_{3}\right)\right) \\
& -\frac{B}{4 D}\left(A_{1}^{2}+A_{2}^{2}+A_{3}^{2}+2 A_{1}\left(A_{2}-B_{3}\right)-2 A_{3}\left(B_{1}-B_{3}\right)-2 A_{2} B_{3}\right. \\
& \left.+\left(B_{1}-B_{2}-B_{3}\right)\left(B_{1}+B_{2}-B_{3}\right)\right), \\
R_{1223}= & \frac{1}{2}\left(B_{22}-B_{12}-B_{23}+A_{13}\right) \\
+ & \frac{A+B}{4 D}\left(A_{2}\left(B_{2}+B_{3}-B_{1}\right)-\left(2 B_{3}-A_{1}\right)\left(2 B_{2}-A_{3}\right)\right) \\
& -\frac{B}{4 D}\left(-A_{1}^{2}+A_{2}^{2}+A_{3}^{2}+2 A_{1}\left(B_{2}+B_{3}\right)+2 A_{2}\left(B_{2}-B_{3}\right)-4 B_{2} B_{3}\right. \\
+ & \left.2 A_{3}\left(B_{3}-B_{1}\right)+\left(B_{1}+B_{2}-B_{3}\right)\left(B_{1}-B_{2}-B_{3}\right)\right), \\
& \\
& \\
&
\end{aligned}
$$




$$
\begin{aligned}
R_{1323} & =\frac{1}{2}\left(B_{23}-B_{33}+B_{13}-A_{12}\right) \\
& +\frac{A+B}{4 D}\left(\left(2 B_{2}-A_{1}\right)\left(2 B_{3}-A_{2}\right)-A_{3}\left(-B_{1}+B_{2}+B_{3}\right)\right) \\
& -\frac{B}{4 D}\left(A_{1}^{2}-A_{2}^{2}-A_{3}^{2}-2 A_{1}\left(B_{2}+B_{3}\right)+2 A_{2}\left(B_{1}-B_{2}\right)+4 B_{2} B_{3}\right. \\
& \left.\left.+2 A_{3}\left(B_{2}-B_{3}\right)-B_{1}+B_{2}+B_{3}\right)\left(B_{1}-B_{2}+B_{3}\right)\right) .
\end{aligned}
$$

The rest of the nonzero components are obtained by the properties

$$
R_{i j k h}=R_{k h i j}, R_{i j k h}=-R_{j i k h}=-R_{i j h k} .
$$

If $\{x, y\}$ is a non-degenerate 2-plane spanned by vectors $x, y \in T_{p} M$, then its sectional curvature is

$$
\mu(x, y)=\frac{R(x, y, x, y)}{g(x, x) g(y, y)-g^{2}(x, y)} .
$$

In this section we obtain some curvature properties of a manifold $(M, g, q)$ on which the following identity is valid

$$
R(q x, q y, q z, q u)=R(x, y, z, u) .
$$

Theorem 3.2. Let $(M, g, q)$ be a manifold with property (15). If a vector $u$ induces a $q$-basis of $T_{p} M$ and $\left\{x, q x, q^{2} x\right\}$ is an orthonormal $q$-basis of $T_{p} M$, then

$$
\mu(u, q u)-\mu(x, q x)=\frac{2 \cos \varphi}{1-\cos \varphi} R\left(x, q x, x, q^{2} x\right),
$$

where $\varphi=\angle(u, q u)$.

Proof. From (15) we find

$$
R\left(q^{2} x, q^{2} y, q^{2} z, q^{2} u\right)=R(q x, q y, q z, q u)=R(x, y, z, u) .
$$

In (17) we substitute $q x$ for $y, q^{2} x$ for $z$ and $x$ for $u$. Comparing the obtained results, we get

$$
R\left(x, q x, q^{2} x, x\right)=R\left(q x, q^{2} x, x, q x\right)=R\left(q^{2} x, x, q x, q^{2} x\right) .
$$


Let $u=\alpha x+\beta q x+\gamma q^{2} x$, where $\alpha, \beta, \gamma \in \mathbb{R}$. Then $q u=-\gamma x+\alpha q x+\beta q^{2} x$. Using the linear properties of the curvature tensor $R$, we obtain

$$
\begin{aligned}
R(u, q u, u, q u) & =\left(\alpha^{2}+\beta \gamma\right)^{2} R(x, q x, x, q x) \\
& +\left(\gamma^{2}+\alpha \beta\right)^{2} R\left(x, q^{2} x, x, q^{2} x\right) \\
& +\left(\beta^{2}-\alpha \gamma\right)^{2} R\left(q x, q^{2} x, q x, q^{2} x\right) \\
& -2\left(\alpha^{2}+\beta \gamma\right)\left(\gamma^{2}+\alpha \beta\right) R\left(x, q x, q^{2} x, x\right) \\
& -2\left(\gamma^{2}+\alpha \beta\right)\left(\beta^{2}-\alpha \gamma\right) R\left(q^{2} x, x, q x, q^{2} x\right) \\
& +2\left(\alpha^{2}+\beta \gamma\right)\left(\beta^{2}-\alpha \gamma\right) R\left(x, q x, q x, q^{2} x\right) .
\end{aligned}
$$

Having in mind (15) and (18), from the latter equality we get

$$
\begin{aligned}
& R(u, q u, u, q u)= \\
& =\left(\left(\alpha^{2}+\beta \gamma\right)^{2}+\left(\gamma^{2}+\alpha \beta\right)^{2}+\left(\beta^{2}-\alpha \gamma\right)^{2}\right) R(x, q x, x, q x) \\
& +2\left(\left(\alpha^{2}+\beta \gamma\right)\left(-\gamma^{2}-\alpha \beta\right)+\left(-\gamma^{2}-\alpha \beta\right)\left(\beta^{2}-\alpha \gamma\right)\right. \\
& \left.-\left(\alpha^{2}+\beta \gamma\right)\left(\beta^{2}-\alpha \gamma\right)\right) R\left(x, q x, q^{2} x, x\right) .
\end{aligned}
$$

Since the $q$-basis $\left\{x, q x, q^{2} x\right\}$ is orthonormal we have

$$
g(u, u)=g(q u, q u)=\alpha^{2}+\beta^{2}+\gamma^{2}, \quad g(u, q u)=\alpha \beta+\beta \gamma-\gamma \alpha .
$$

Due to (5) and (14) we get

$$
\mu(u, q u)=\frac{R(u, q u, u, q u)}{g(u, u) g(q u, q u)-g^{2}(u, q u)} .
$$

We can suppose that $g(u, u)=1$. Then we obtain

$$
\mu(u, q u)=\frac{R(u, q u, u, q u)}{1-\cos ^{2} \varphi}
$$

and

$$
\alpha^{2}+\beta^{2}+\gamma^{2}=1, \quad \alpha \beta+\beta \gamma-\gamma \alpha=\cos \varphi .
$$

The latter two equations imply

$$
\begin{aligned}
\cos ^{2} \varphi+\cos \varphi & =\left(\alpha^{2}+\beta \gamma\right)\left(\gamma^{2}+\alpha \beta\right)+\left(\gamma^{2}+\alpha \beta\right)\left(\beta^{2}-\alpha \gamma\right) \\
& +\left(\alpha^{2}+\beta \gamma\right)\left(\beta^{2}-\alpha \gamma\right), \\
1-\cos ^{2} \varphi & =\left(\alpha^{2}+\beta \gamma\right)^{2}+\left(\gamma^{2}+\alpha \beta\right)^{2}+\left(\beta^{2}-\alpha \gamma\right)^{2} .
\end{aligned}
$$

Then, using (19) and (20) we obtain (16). 
Theorem 3.3. Let $(M, g, q)$ be a manifold with property (15). If a vector $u$ induces a $q$-basis of $T_{p} M$ and $\left\{x, q x, q^{2} x\right\}$ is an orthonormal $q$-basis of $T_{p} M$, then for $x, u, y \in T_{p} M$ with $\angle(y, q y)=\frac{2 \pi}{3}$ the following equality is valid

$$
\mu(u, q u)=\frac{1+2 \cos \varphi}{1-\cos \varphi} \mu(x, q x)-\frac{3 \cos \varphi}{1-\cos \varphi} \mu(y, q y),
$$

where $\varphi=\angle(u, q u)$.

Proof. In (16) we substitute $y$ for $u$ and because of the condition $\angle(y, q y)=\frac{2 \pi}{3}$ we get

$$
R\left(x, q x, x, q^{2} x\right)=\frac{3}{2}(\mu(x, q x)-\mu(y, q y)) .
$$

Taking into account the last result and (16), we get (21).

Proposition 3.4. Let $(M, g, q)$ be a manifold with property (15). If a vector $u$ induces a q-basis of $T_{p} M$, then the sectional curvatures of the 2-planes $\{u, q u\},\left\{q u, q^{2} u\right\}$ and $\left\{q^{2} u, u\right\}$ are equal.

Proof. In (17) we substitute $u$ for $x, q u$ for $y, u$ for $z$ and $q u$ for $u$. Then we get

$$
R(u, q u, u, q u)=R\left(u, q^{2} u, q u, q^{2} u\right)=R\left(q u, q^{2} u, q u, q^{2} u\right) .
$$

From (15), (14) and (22) for the sectional curvatures of the 2-planes of a $q$-basis $\left\{u, q u, q^{2} u\right\}$ we obtain $\mu(u, q u)=\mu\left(u, q^{2} u\right)=\mu\left(q u, q^{2} u\right)$.

Proposition 3.5. The property (15) of the manifold $(M, g, q)$ is equivalent to the conditions

$$
R_{1212}=R_{1313}=R_{2323}, \quad R_{1213}=R_{1323}=R_{1223},
$$

where $R_{i j k h}$ are the local components of the curvature tensor $R$ of type $(0,4)$. Proof. The local form of (15) is

$$
R_{t s l m} q_{i}^{t} q_{j}^{s} q_{k}^{l} q_{h}^{m}=R_{i j k h} .
$$

From (24) and (44) we find

$$
\begin{array}{ll}
R_{1212}=R_{2323}, & R_{1313}=R_{2121}, \\
R_{2321}=R_{1213}, & R_{2331}=R_{1223}, \\
R_{2131}=R_{1323}, & R_{3131}=R_{2323},
\end{array}
$$

which implies (23).

Vice versa, (25) follows from (23). Having in mind (44), we get (24). 


\section{A necessary and sufficient condition for $\nabla q=0$}

The covariant derivative of the structure $q$ with respect to $\nabla$ of $g$ is [10]

$$
\nabla_{i} q_{j}^{h}=\partial_{i} q_{j}^{h}+\Gamma_{i t}^{h} q_{j}^{t}-\Gamma_{i j}^{t} q_{t}^{h} .
$$

Let the structure $q$ be parallel with respect to the Riemannian connection $\nabla$ of our manifold $(M, g, q)$, i.e. $\nabla q=0$. Then from (4) and (26) follows

$$
\begin{array}{lll}
\Gamma_{11}^{3}=\Gamma_{32}^{1}, & \Gamma_{11}^{2}=\Gamma_{32}^{2}, & \Gamma_{11}^{3}=\Gamma_{32}^{3}, \\
\Gamma_{21}^{2}=\Gamma_{33}^{2}, & \Gamma_{21}^{1}=\Gamma_{33}^{1}, & \Gamma_{21}^{3}=\Gamma_{33}^{3}, \\
\Gamma_{22}^{1}=\Gamma_{13}^{1}, & \Gamma_{22}^{2}=\Gamma_{13}^{2}, & \Gamma_{22}^{3}=\Gamma_{13}^{3} .
\end{array}
$$

Using (1), (12) and (27), we obtain

$$
A_{1}=-B_{1}+B_{2}+B_{3}, A_{2}=B_{1}-B_{2}+B_{3}, A_{3}=B_{1}+B_{2}-B_{3} .
$$

The system (28) has the matrix form

$$
\left(A_{1}, A_{2}, A_{3}\right)=\left(B_{1}, B_{2}, B_{3}\right)\left(\begin{array}{ccc}
-1 & 1 & 1 \\
1 & -1 & 1 \\
1 & 1 & -1
\end{array}\right) .
$$

Therefore, we have

$$
\operatorname{grad} A=\operatorname{grad} B\left(\begin{array}{ccc}
-1 & 1 & 1 \\
1 & -1 & 1 \\
1 & 1 & -1
\end{array}\right)
$$

where $\operatorname{grad} A$ and $\operatorname{grad} B$ are gradients of the functions $A$ and $B$.

Vice versa, let (29) be valid. Then (1) and (12) imply

$$
\begin{aligned}
& \Gamma_{11}^{1}=\Gamma_{12}^{2}=\Gamma_{13}^{3}=\Gamma_{22}^{3}=\Gamma_{23}^{1}=\Gamma_{33}^{2}=\frac{1}{2 D}\left(A A_{1}+B\left(B_{2}-3 B_{1}+B_{3}\right)\right), \\
& \Gamma_{11}^{3}=\Gamma_{12}^{1}=\Gamma_{13}^{2}=\Gamma_{22}^{2}=\Gamma_{23}^{3}=\Gamma_{33}^{1}=\frac{1}{2 D}\left(A A_{2}+B\left(B_{3}-3 B_{2}+B_{1}\right)\right), \\
& \Gamma_{11}^{2}=\Gamma_{12}^{3}=\Gamma_{13}^{1}=\Gamma_{22}^{1}=\Gamma_{23}^{2}=\Gamma_{33}^{3}=\frac{1}{2 D}\left(A A_{3}+B\left(B_{1}-3 B_{3}+B_{2}\right)\right) .
\end{aligned}
$$

Now, we verify that $\Gamma_{i t}^{h} q_{j}^{t}=\Gamma_{i j}^{t} q_{t}^{h}$ is valid. That means $\nabla_{i} q_{j}^{s}=0$, i.e. $\nabla q=0$.

Thus, it is valid the following

Theorem 4.1. The structure $q$ is parallel with respect to the Riemannian connection $\nabla$ of $g$ on a manifold $(M, g, q)$ if and only if the condition (29) is valid. 


\section{Example of a manifold $(M, g, q)$}

Let $(M, g, q)$ be a manifold with a metric $g$ determined by (11) and

$$
A=2 X^{1}, B=2 X^{1}+X^{2}+X^{3},
$$

where

$$
2 X^{1}+X^{2}+X^{3}>0, X^{2}+X^{3}<0 .
$$

Obviously, the condition (22) is satisfied. Due to (13), (30) and Proposition 3.1 we obtain

$$
\begin{aligned}
& R_{1212}=R_{1313}=R_{2323}=\frac{2 X^{1}+X^{2}+X^{3}}{\left(X^{2}+X^{3}\right)\left(6 X^{1}+2 X^{2}+2 X^{3}\right)}, \\
& R_{1213}=R_{1323}=R_{1223}=0 .
\end{aligned}
$$

We check directly that the conditions (23) are valid, but the condition (29) for the functions $A$ and $B$ is not valid.

Therefore, it is valid the following

Proposition 5.1. The manifold $(M, g, q)$ with a metric $g$ defined by (30) has the following properties:

(i) The curvature identity (15) is valid.

(ii) The structure $q$ is not parallel with respect to the Riemannian connection $\nabla$ of $g$.

(iii) $M$ is not a flat manifold.

\section{References}

[1] Borisov, A., Ganchev, G.: Curvature properties of Kaehlerian manifolds with B-metric. Math. Educ. Math., Proc of 14th Spring Conf. of UBM, Sunny Beach, 220-226 (1985)

[2] Gribachev, K., Mekerov, D., Duelepov, G.: On the geometry of almost B-manifolds Compt. Rend. Acad. Bulg. Sci. 38, 563-566 (1985)

[3] Gribachev, K., Duelepov, G.: On the geometry of the normal generalized B-manifolds. Plovdiv Univ. Sci. Works - Math. 23, 157-168 (1985)

[4] Davis, P. R.: Circulant matrices. John Wiley, New York (1979) 
[5] Dzhelepov, G., Dokuzova, I., Razpopov, D.: On a threedimensional Riemannian manifold with an additional structure. Plovdiv Univ. Sci. Works - Math. 38, 17-27 (2011)

[6] Reyes, E., Montesinos, A., Gadea, P.M.: Connections making parallel a metric $\left(J^{4}=1\right)$-structure. An. Sti. Univ. "Al. I. Cuza" 28, 49-54 (1982)

[7] Stanilov, G., Filipova, L.: About the circulate geometry, In: Proceedings of XVI Geometrical Seminar, Vrnjachka Banja, 96-102 (2010)

[8] Yano, K., Ishinara, S.: Structure defined by $f$ satisfying $f^{3}+f=$ 0. Proc.US-Japan Seminar in Differential Geometry., Kyoto, 153-166 (1965)

[9] YANO, K.: On a structure defined by a tensor field of type $(1,1)$ satisfying $f^{3}+f=0$. Tensor, 99-109 (1963)

[10] Yano, K.: Differential geometry on complex and almost complex spaces. Pure and Applied Math. 49, New York, Pergamont Press Book, (1965)

Iva Dokuzova

Department of Geometry

FMI, University of Plovdiv

Plovdiv, Bulgaria

dokuzova@uni-plovdiv.bg 